are so little employed by regular practitioners- the cauterization of the back of the neck by moxas or by the red-hot iron.

Other affections very often have the same characteristic fea tures as epilepsy, as regards their production. If I had time, I could relate a very large number of facts to profe that, much more frequently than might be imagined by most of my hearers, the various forms of insanity, of vertigo, of hallncinations, and of illusions, and also extasis, catalepsy, hysteria, chorea, hydrophobia, tetanus, local cramps, and even the general paralysis connected with insanity, may be due to irritations starting from a centripetal nerve, and frequently slightly felt, or even unfelt; and that the suppression of these irritations may promptly cure the patient, just as in cases of epilepsy. Instead of a description of this kind of affection, $I$ will give here a case which is full of interest, and which I owe to the kindness of Mr. Campbell de Morgan, who had received it from the late Mr. Standert, of Taunton, who, according to Mr. de Morgan, was one of the most original thinkers and best surgeons of his day :-

CASE 44. - On rising in the morning, a lad, fourteen years old, was heard by his father making a great disturbance in his bed-room, who, rushing in to know the cause, found his son in his shirt, violently agitated, speaking incoherently, and breaking to pieces the furniture. Mr. - caught the lad in his arms, and threw him back on the bed, when he at once became com. posed, but did not seem conscious of the mischief he had done. He said that on getting out of bed he had felt something odd, but that he was very well, and thought that he might have had a frightful dream, although he could not recollect it. was immediately sent for, and the lad ordered to remain in bed until I had seen him. About five hours after, I found the lad lying in bed, reading some amusing book; his tongue clean, pulse regular, countenance caim and cheerful. He said he was quite well, and wished to get up, but that his father had ordered him to remain in bed until I had seen him. I was informed, before I went up to his bed-room, that the lad had never before been heard to complain of disturbed dreams, or walked in his sleep, or exhibited any epileptic symptoms, and that his general health had been good, and all his functions re gular. Finding the patient free from any apparent disease, and that he had eaten with good appetite, and no disturbance, his usual breakfast, I desired him to get up. When, sitting up in his bed, he drew on his stockings; but on putting his feet on the floor and standing up, his countenance instantly changed, the jaw became violenty convulsed, and he was about to rush forward, when I seized and pushed him back on the bed. $\mathrm{He}$ was at once calm, but looked surprised, and asked what was the matter with him. He assured me that he had felt no pain had slept well, but that he "felt odd" when he stood up. I found that he had been fishing on the preceding day, and, having entangled his line, had taken off his shoes and stockings, and waded into the river to disengage it; but he said he had not cut or scratched his feet or met with any other accident. To ascertain this point, I made him draw off his stockings, and examined his legs minutely. Not the slightest scratch or injury could be seen; but on holding up the right great toe with my finger and thumb to examine the sole of that foot, the leg was drawn up, and the muscles of the jaws were suddenly conviulsed, and on releasing the toe these effects instantly ceased. I then closely inspected the toe. The nail was perfect; there was not the least swelling or redness in the surrounding parts, nor any tenderness or uneasiness felt when I compressed the toe laterally, or moved it, held thus, in any direction; but on the bulb of the toe, nearly at the point where the circumgyrations of the cuticle centre, there was a rery small elevation, as if a bit of gravel, less than the head of a small pin, had been there pressed in beneath the cuticle. There was not the least redness on this spot, nor any sensation or effect produced by passing my fiuger over its surface; but on compressing it with my finger and thumb agairst the nait very cautiously, a slight convulsion instantly ensued. I asked the patient if anything pricked him ? He said " No, but something mude him feel ve"y odd." On examining the part well with a pocket lens, no scratch or puncture of the cuticle could be discerned. I then with a pair of scissors included and snipped away the slightly elevated part, but not so deeply as to denude the cutis beneath. In the bit of cuticle thus removed I expected to find some point of a thorn or particle of sand, but could not detect anything of the kind. I then pressed the toe in every direction; the strange sensation was gone, and never returned.

I do not know that any member of the patient's family had ever been under treatment for insanity, bat two of his uncles and $I$ believe an aunt were suiciles, and the patient himself, many years afterwards, was "found drowned" by the cautious verciict of an inquest.

\section{ON SCARLATINA AND ITS TREATMENT.}

\section{Br E. BISHOP, M.D., Deronport.}

If we would have a science, and the art founded thereon, to become more and more pure, we must direct our studies in the way which our present acquaintance with the science points ont to us. If the way be one of theory, we must theorize; if it be one of practice and observation, we must observe and investigate. Medicine is essentially a science of facts. The store of facts is gradually accumulating, and has already, indeed, increased to such an extent as to render the teaching necessarily more and more practical, and to show that things, not words en masse, form the material with which the practitioner's mind must be supplied. The more medicine is made to partake of the real, the more prominent and brightly will it shine.

The foregoing I saw in print some time since, and the remarks appear applicable to the following. It is desirable that contributions to the medical press, however trifling they may appear to some, should be published, especially respecting any new or successful treatment in serious diseases. It is the duty of the practitioner to record his method of treating disease, even if it does not appear strictly orthodox, or in accordance with the prescribed methods. If we had to rest entirely upon the authority of even our best authors, we should fall miserably short; for the numerous opinions given as to the nature and treatment of this and other diseases are so contradictory and conflicting as to mislead the matured as well as the juvenile practitioner. Or, to quote the language of Dr. Gilmour, " My young brethren in medicine must not trust implicitly to what they read in books; many of them are truly valuable and trustworthy, but others (and of these there is a large number) are written to suit a purpose, and contain trash,"

I beg to state as briefly as possible the treatment I have adopted in this disease during the last nine months. There is nothing original in it, so far as it relates to myself. I know friends who have tried it with marked success. Scarlatina has been rife in this town for the last nine months, and has proved fatal to a large number of children belonging to all classes of society. In many families, one, two, and even three, have succumbed to it. I applied to the registrar for the exact number of deaths from this disease in the three quarters ending March, June, and September, 1858, and he has been kind enough to supply me with a return, by permission of the Registrar-General. In the first quarter of the present year the deaths from scarlatina reached 23 ; in the second, 44 ; and in the third, 27, in children under ten years of age.

In fifty-one cases of scarlatina scattered over the town, in children varying from two to ten years of age, my plan of treatment has been tonics from the commencement-(i. e., from my first visit)-either the citrate of iron, or the tincture of the sesquichloride, in the usual full doses; and I have had every reason to be satisfied with the result, having lost but one case. I made no difference in the plan of treatment even when serious complications presented. In many the fever was intense, the inflammation of the throat severe, and pain in swallowing very considerable. Four children in one house, in Cannon-street, had scarlatina anginosa in an aggravated form, being attended with an acrid discharge from the ears and nostrils. In one case deafness has remained nearly permanent. I entirely discarded the application of strong caustics to the throat and tonsils, which many years' experience has taught me is injurious in very young children, and calculated to do more harm than good, to say nothing of the injury and difficulty attending the operation. External applications to the throat I found most beneficial-either the compound camphor liniment, oil and hartshorn, or turpentine sprinkled on a strip of flannel, previously wrung ont of hot water, and applied several times during the twenty-four hours. Inhaling the steam of hot water gave much relief, as it generally does. The children I have been called upon to treat, have belonged to the poorer class; the diet necessarily simple; in severe cases, broths, beeftea, millk, jelly, and wine were recommended, and procured if possible. One gratifying result in the treatment of scarlatina with iron, as far as my experience carries me, is that the children, with few exceptions, escaped that serious and frequent sequel-anasarca.

I have seen two cases of diphtheria following scarlatina during the epidemic, in children four and six years of age. The first child had been convalescent a week or ten days, and, I must confess, I could not understand the cause of the relapse. 573 
After a few days, I suspected diphtheria; the child would never allow me nor the parents to examine the state of the throat, although rough usace was resorted to more than once or twice. During the time it suffered extreme prostration, and was supported by wine and beef-tea; it also took a mixture containing the tincture of the sescuichloride of iron. This child ultimately coughed up the membrane characteristic of diphtheria. A tit of vomiting and coughing came on at a time when it appeared beyond hope; but when the membrane was released, the child was relieved, and gradually rallied.

A remarkable case under mv care was that of a boy aged four years, belonging to the Royal Naval and Military Free Schools. I first saw him twelve weeks ano from the date of this communication; he had then scarlatina anginosa, from which he recovered sufficiently to enjoy a walls. About a fortnight after his recovery his mother requested me to see him, as he had (to use her own words) "pimples coming out over his face and body." On visiting him this proved to be variola discreta. He had been successfully vaccinated, judging from the cicatrices in his arm. The little fellow suffered severely; he had not regained his strength from the debilitating consequences of the previous illness. He was kept up by wine, beef-tea, ammonia, and bark, as maturation of the pustules went on but slowly. He recovered from this attack, but not sufficiently to return to school, when I was requested to visit him, as he had hooping-cough, which was and is now epidemic in this town; and this being complicated with pneumonia, terminated his existence a few days ago. There was something remarkable in the fact of this child having three of the most serious and fatal diseases of childhood in the short period of three months. One little girl who was in the habit of going to the house of the deceased is now under treatment for variola.

I trust the tonic treatment of scarlatina with iron may have a trial elsewhere, and prove as efficacious and successful as it has been with me. I conceive it is far better to prove by facts than to judge and condemn without a trial, simply because the treatment does not harmonize with the doctrine laid down by our popular authors and preceptors.

The population of the borough of Devonport in 1851, as set forth in the Government Returns, was 38,180. The total number of deaths from all causes from Midsummer 1857 to Midsummer of the present year, exceeded 1000 . This, to me, appears high, and well deserving the attention of every medical practitioner of the place. My experience of Devonport scarcely extends over a period of two years, still I have seen enough to satisfy me that overcrowding, with its concomitant dirt and vitiated atmosphere, and the abominable and disgustingly filthy state of many courts and alleys, produce increase of disease of every kind. Many courts the sunshine never enters, and are notoriously tainted; they are, literally speaking, "fever nests" for the poor mariner who has enjoyed two or three years of healthy labour at sea, and who pitches, perhaps, on a locality where disease finds a resting-place, and is constantly dealing death and destruction to those around. Last year I addressed a lengthy communication to the publio press on the subject of sanitary improvement, and the (then) probable approach of cholera to our shores. Considering that this place is becoming one of the largest and most important naval and military stations in the south of England, an excess of disease of any kind is a matter which deeply concerns every inhabitant; and available means should be suggested to the several officials to lower the rate of mortality by improving the sanitary condition. I could enlarge on this subject; but at present $I$ will merely observe that the heavy mortality from scarlatina and types of low fever generally prevalent throughout the town, are in a great degree dependent on causes capable of being modified or counteracted; but I fear that, under existing arrangements, the matter will not meet with that careful investigation which it deserves. The density of population is greater than that of any other place I am acquainted with; and when any epidemic prevails, it is greatly aggravated in consequence of the various evils specified above. How far the mortality from scarlatina in young children, which has amounted to nearly one hundred in nine months, is dependent on causes capable of being lessened or counteracted, is a question deserving our consideration; and though it is impossible to prevent infectious disorders coming amongst us, there can be no doubt that they may be modified by means which we have under our control, if only put into operation and exercised judiciously. However objectionable it may be to some, it is a fact prominent in the minds of impartial observers and in indubitable records, that Devonport, in its present state, is not salubrious as a residence; and $I$ have no doubt that some of my professional friends long resident here could indicate causes which contribute to produce the degenerating state of health and the barrier o sanitary improvement.

November 22 nd, 1858 .

ON THE

\section{COMMUNICABILTTY OF CHOLERA.}

\section{BY PHILIP B. AYRES, M.D. Lond.} SCPERINTENDENT OF QDARANTINE, MAUBITITS.

THE mode of propagation of cholera has of late years formed a subject of warm dispute between two parties in the medical profession-the contagionists and the non-contagionists, as they are usually termed. While in India the capability of communication of this fearful disease is almost universally denied, opinion has been much divided amongst European practitioners, and the questions, first, whether choleta is propagated from individual to individual; and secondly, if communicated at all, whether the poison finds entrance to the system by the skin, the pulmonary mucous membrane, or that of the alimentary canal, - have been largely discussed.

The question of the communicability of disease is one usually surrounded by great aifficulties, because the propagation of disease has necessarily been studied in large communities, and the only opportunities for establishing the fact of communication have been where the epidemic has made its appearance in small and remote villages, in which the movements of the few inhabitants can be most accurately traced. In larger towns, the movements of an individual can scarcely be followed, as is daily shown by the extreme difficulty with which the police track $a$ criminal, and get up the evidence against him, so as to insure conviction.

The most striking example of the introduction of cholera into an island, previously and at other times perfectly exempt from cholera as an endemic disease, is afforded by the island of Mauritins, separated from the nearest continent by at least a thousand miles. Mauritius, since the abolition of slavery, has wholly depended for its labour on the introduction of coolie labourers from Incia, who are engaged there for a term of five years, with a free passage to their native country on the expiration of the term. The coolies are collected at Calcutta or Madras, where cholera is almost always prevalent, and brought to Mauritius after an average passage of twenty to thirty days from the latter, and thirty-five to fifty days from the former port. As may be anticipated, cholera not unfrequently makes its appearance to a greater or smaller extent amongst the coolie labourers, especially at the commencement of the voyage, but usually wears itself out long before the voyage is completed. Sometimes, however, from causes not easily to be explained, the disease continues to find victims until the approach to Mauritius; and the inhabitants of the island, having suffered from two fearful epidemics in $18: 4$ and 1856 , precautionary measures have been insisted on and accorded by the Government, and rigid quarantine is now established.

Without making any positive statement as to the mode of introduction of the disease into Mauritius in 1854 and 1856 , it is an undonbted fact that the commencement of these epidemics was almost simultaneous in each year with the arrival of in fected ships at Port Louis. The recent report of a committee of the Legislative Council appointed " to inquire into the probable cause or causes of the recent outbreak of cholera in the island of Mauritius," however, positively attributes the outbreak to commmication with the coolies of two infected ships. The question of quarantine, especially as regards its power of excluding infectious or contagious diseases, is one now seriously discussed in Europe. As quarantine forms a serious impediment to mercantile transactions and individual locomotion, the mercantile community is universally opposed to it, and, aided by the old Board of Health, has endeavoured to throw discredit on its powers of prevention. In the opposition to quarantine, the argument has, for the most part, rested on its inefficient or negligent performance, or on the attempt to prove that certain diseases-cholera, yellow fever, typhus, \&c. - are not infectious or communicable. The brief notice I am about to give of a recent quarantine at Mauritins will, I flatter myself, show in dubitably that cholera is a communicable disease; and that a 